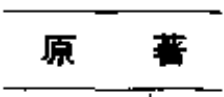

$$
\begin{aligned}
& \text { 大腸ポリープについて；瘿合併例に } \\
& \text { 打若苦の知見 }
\end{aligned}
$$

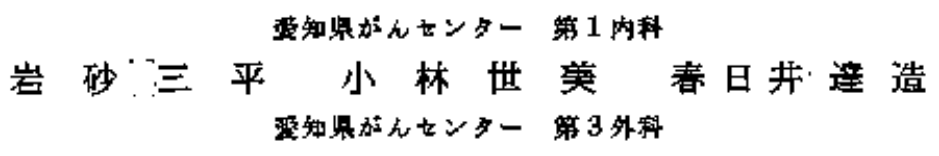

$$
\begin{aligned}
& \text { 加丵 王 干 }
\end{aligned}
$$

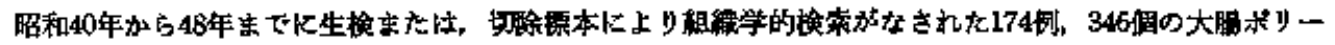

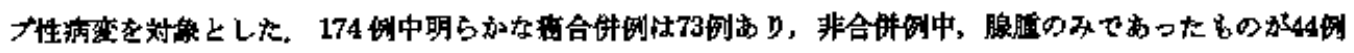

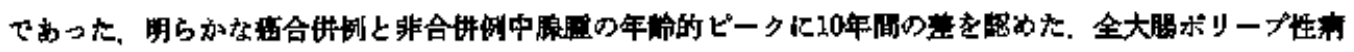

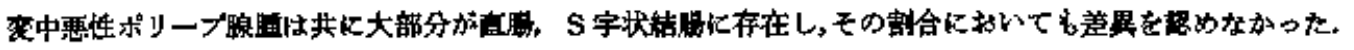

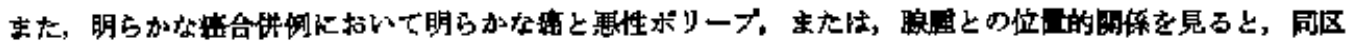

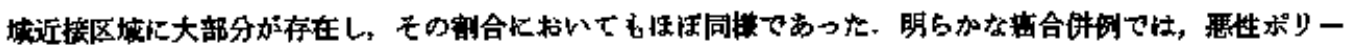

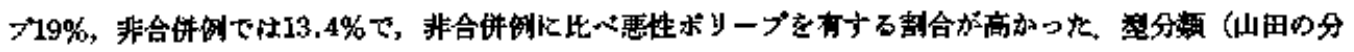

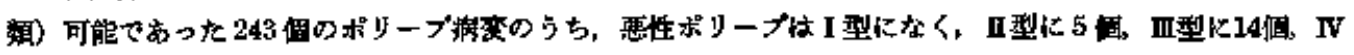

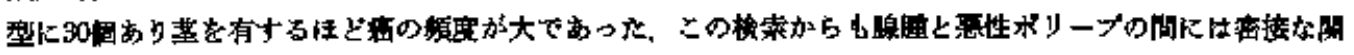
保があることが推赛される。

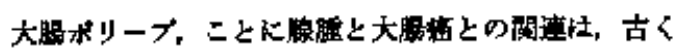

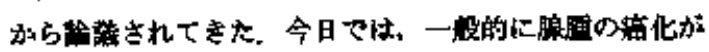

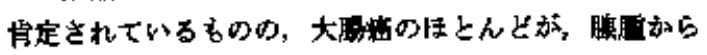

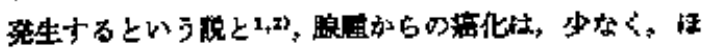

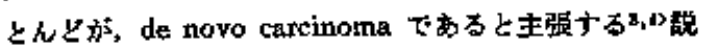
があり，末だ，䊑险に到っていないの放状である、大

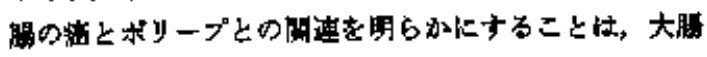

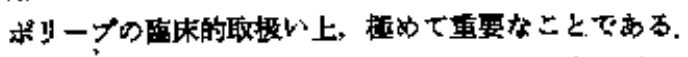

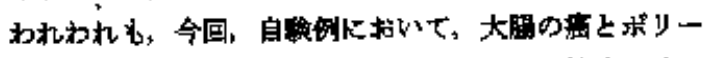

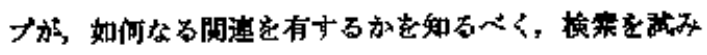
たので, 著干の哮察加えて報告する。

\section{悋}

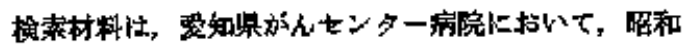

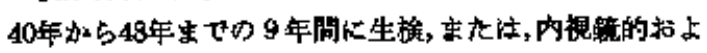

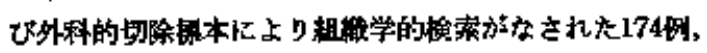

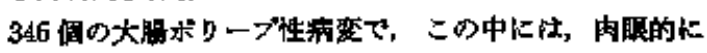

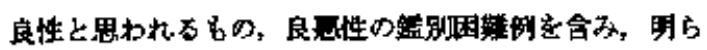

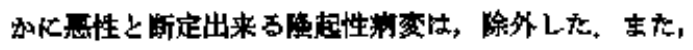
大䯈ポリボージス。除外した.

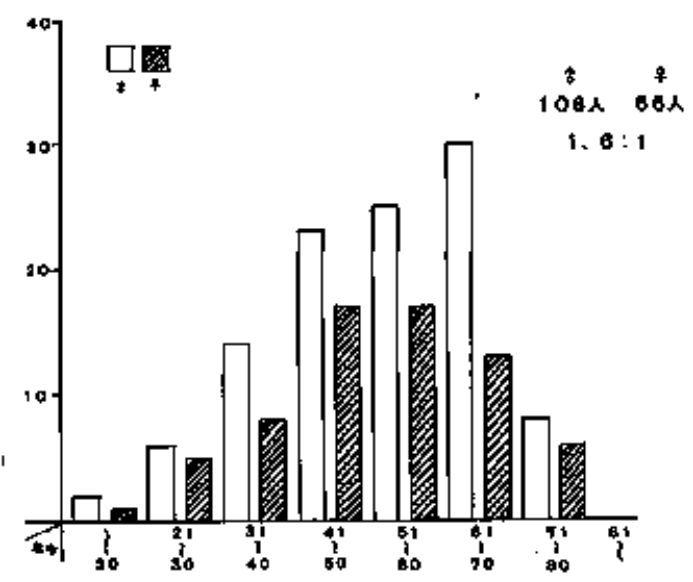

图 1 性别年䭛叧有

\section{成 渵}

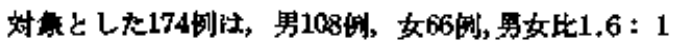
であり，60歳代をビークとする年脑分布を示した(图 1).

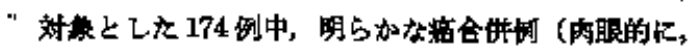

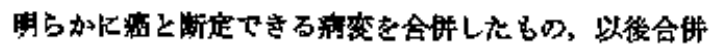

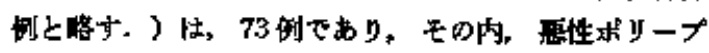
(Carcinoma in adenoma 


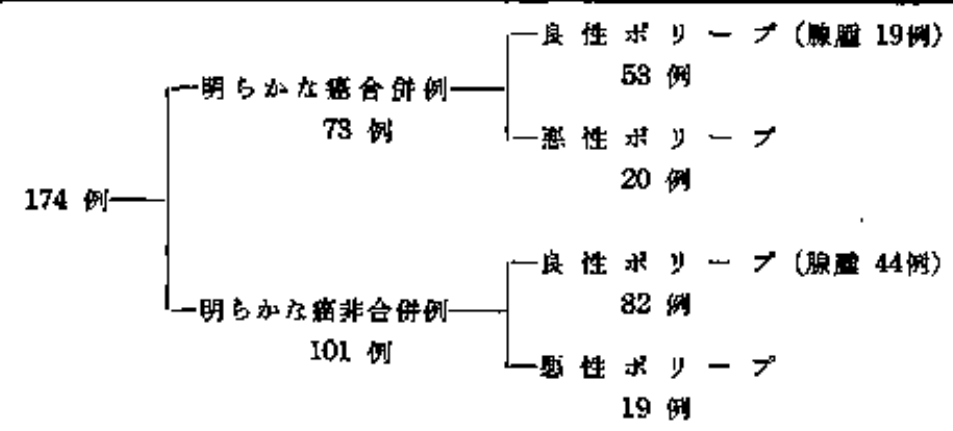

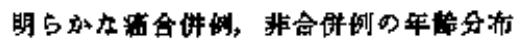
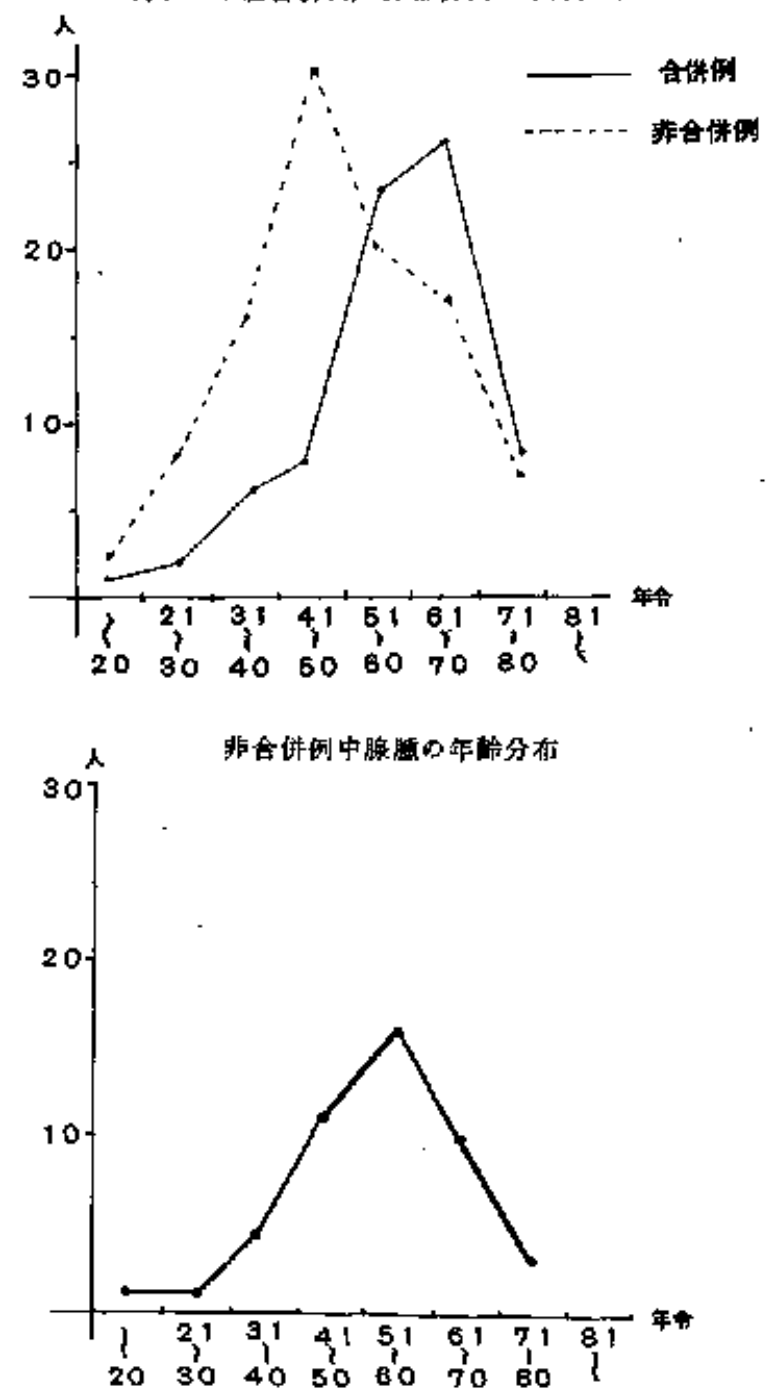

图 2

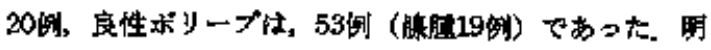

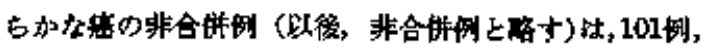

その内，思性ポリープ19湖，良性ボリーブは，62例（脉

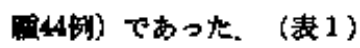

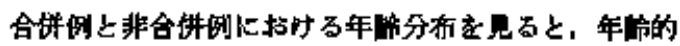
ピークが, 合併例では, 61 70建に, 非合併例で恃, 41

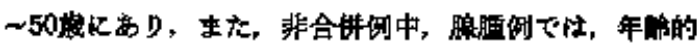

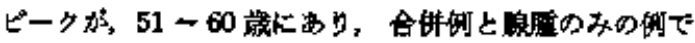

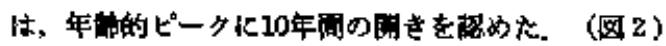

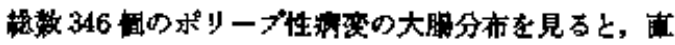

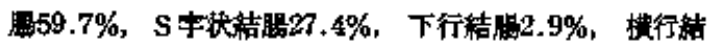

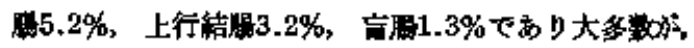

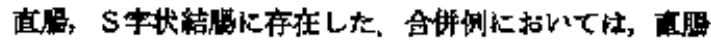

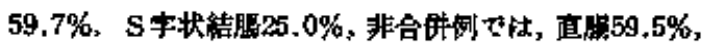

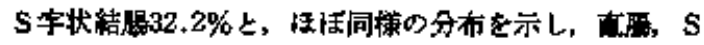

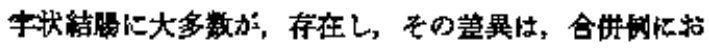

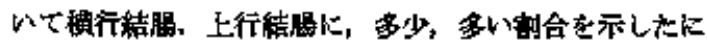
ナざかかった（表 2 )

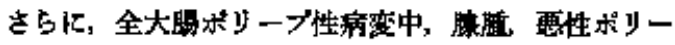

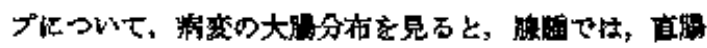
$45.5 \%$ ，S宇状結盟36.6\%を示し，恶性ボリープに掠い て

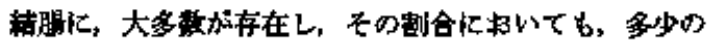

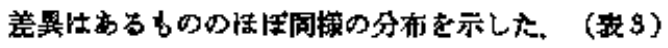

合併倒について，明らかな音と良性ポリーブ、ことに

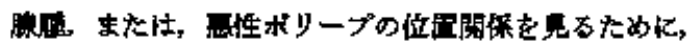

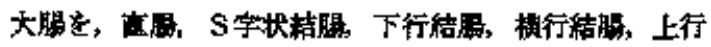

\begin{tabular}{|c|c|c|c|}
\hline & 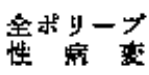 & 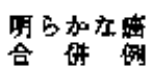 & 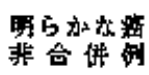 \\
\hline 眦 & $59.7 \%$ & $59.7 \%$ & E9.5\% \\
\hline 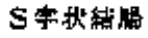 & 27.4 & 25.0 & 32.2 \\
\hline 下行緌盟 & 2.9 & 1.6 & 4.8 \\
\hline 枓行結时 & 5.2 & 7.1 & 2,4 \\
\hline 上行結軻 & 3.2 & 4.9 & $0 . B$ \\
\hline 亩 & 1.3 & 1.6 & 0.8 \\
\hline
\end{tabular}

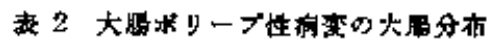




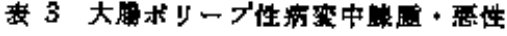
ボりーブの大路分布

\begin{tabular}{|c|c|c|}
\hline & 腺 & 要比ボリーブ \\
\hline 㳻 & $45.5 \%$ & $52.8 \%$ \\
\hline 5 乎状桔明 & 36,6 & 39.8 \\
\hline 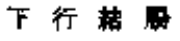 & 5.7 & 1.9 \\
\hline 磁行萧 & 5.7 & 5.7 \\
\hline 上枌䒜 & 5.7 & 0 \\
\hline 浣 & 1.6 & 0 \\
\hline
\end{tabular}

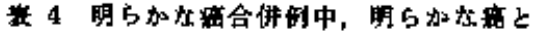

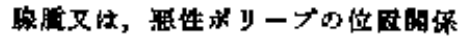

\begin{tabular}{|c|c|c|c|}
\hline$\cdot$ & 膰胵 & 昰性がリーブ & 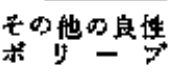 \\
\hline 网区 域 & $52.4 \%$ & $56.3 \%$ & $88.9 \%$ \\
\hline 近转区域 & 44.4 & 40.6 & 10,0 \\
\hline 徒需区塔 & 3.2 & 3.1 & 1.0 \\
\hline
\end{tabular}

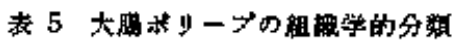

\begin{tabular}{|c|c|c|c|}
\hline & 恶珄ホ⿺ & 素 & ₹ 9 他 \\
\hline $\begin{array}{c}\text { 全术yーブ } \\
\text { (346 调) }\end{array}$ & $16.8 \%$ & $39.3 \%$ & $44.6 \%$ \\
\hline 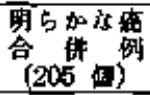 & 19.0 & -35.1 & 45,9 \\
\hline 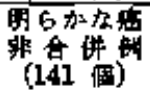 & 13.4 & 43.9 & 42.5 \\
\hline
\end{tabular}

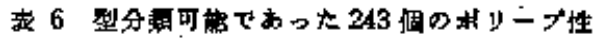

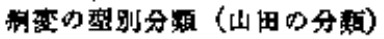

\begin{tabular}{|c|c|c|c|c|}
\hline & I 보 & II 㤠 & III 烈 & IV 㤠 \\
\hline 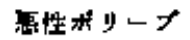 & 0 & 5 & 14 & 30 \\
\hline 䘑 & 10 & 31 & 33 & 34 \\
\hline$\nvdash \quad \infty$ & 9 & 98 & 26 & 13 \\
\hline
\end{tabular}

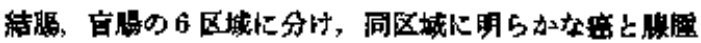

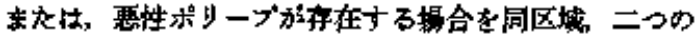

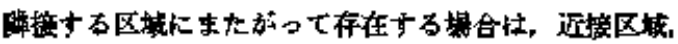

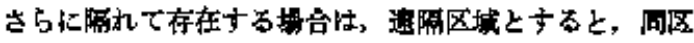

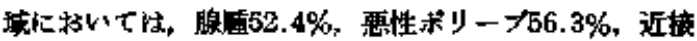

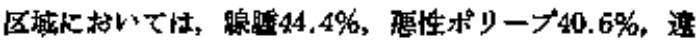

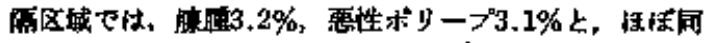
㴍の分布を示した，事た，他の良性ボリーブ性，約90\%

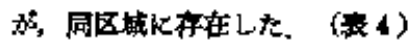

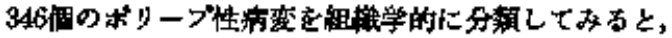

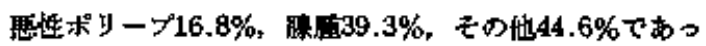

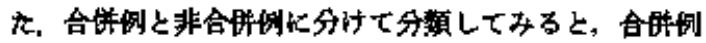

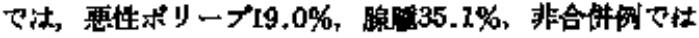

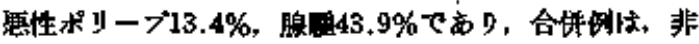

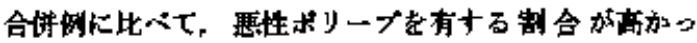
大. (共5)

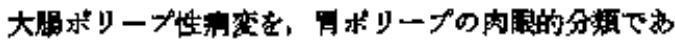
万山田の分類に㷋ってタけてみると, 型分粼可能であっ

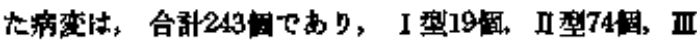

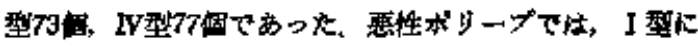

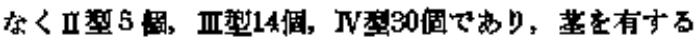

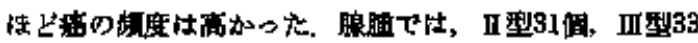

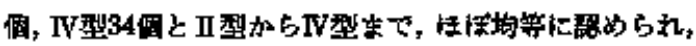

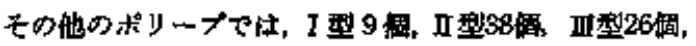

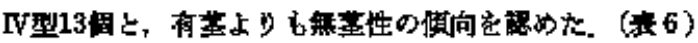

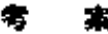

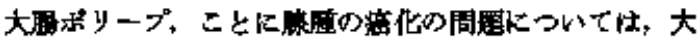

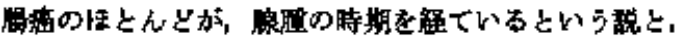

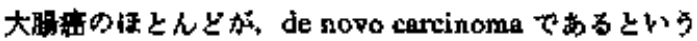

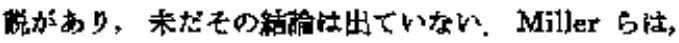

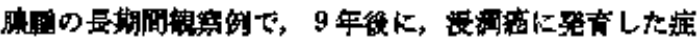

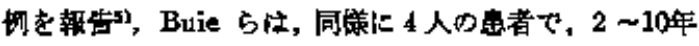

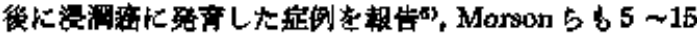

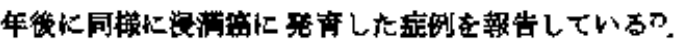

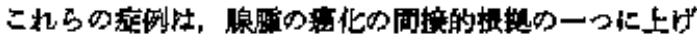

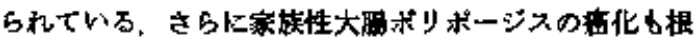

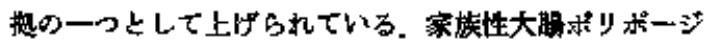

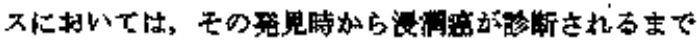

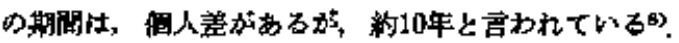

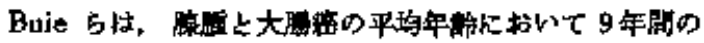

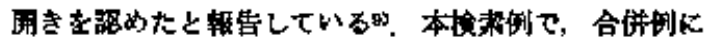

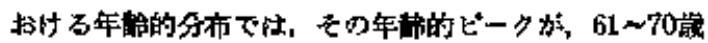

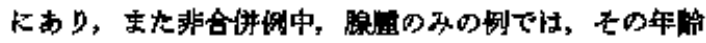

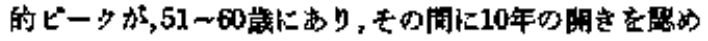

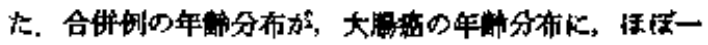

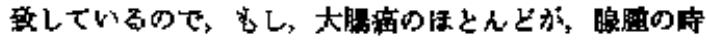

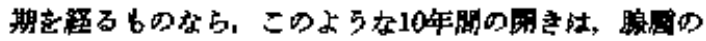

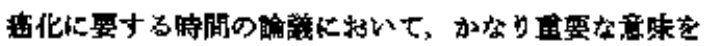
持つと新志。

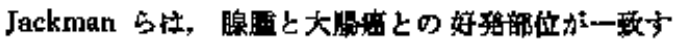

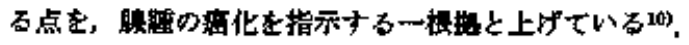

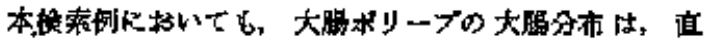

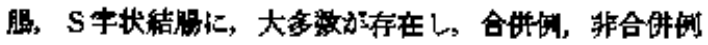

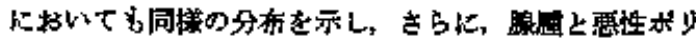

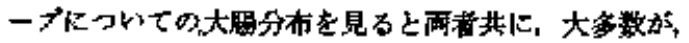

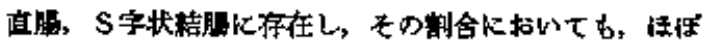

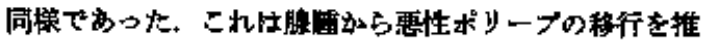


定するに都合のよい毫宾である。

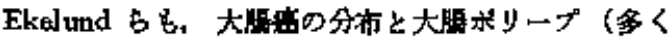

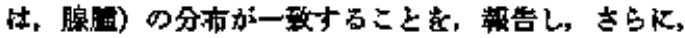

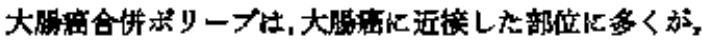
存在ナることし報告しているリ゙. 虾，本邦にわいて

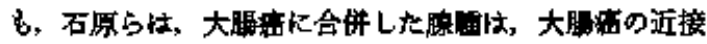

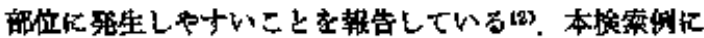

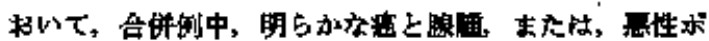

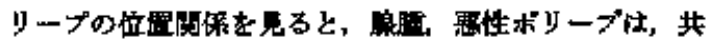

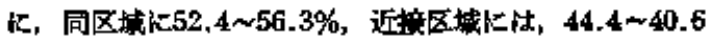

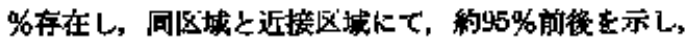

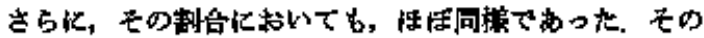
他のポリープ（主仅化生性打よび遗形性ポリープ） は， 同区城，88，9\%方存在し，石原らの化生性ポリーブは，

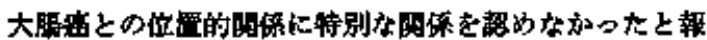

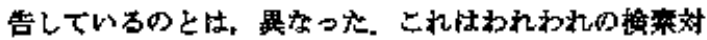
象となったポリーブ性放变中，その他のポリープでは，

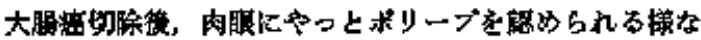

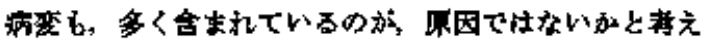
t.

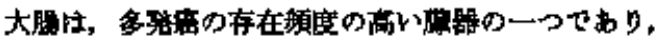

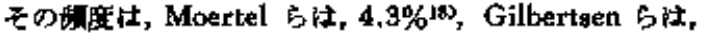

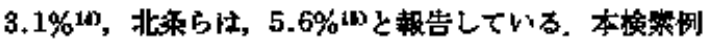

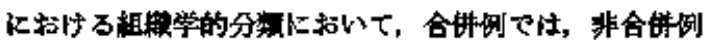

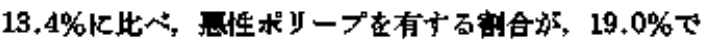

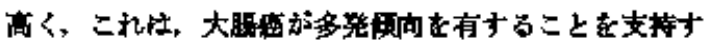

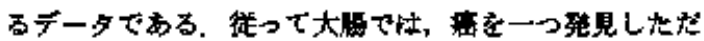

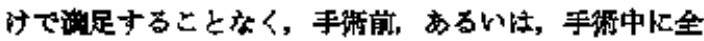

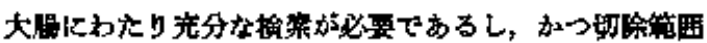

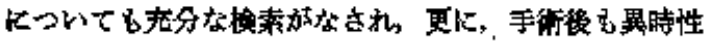

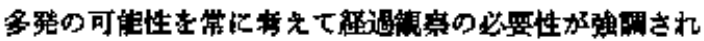
3.

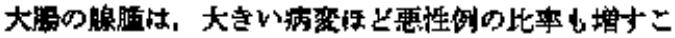

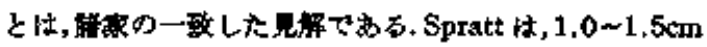

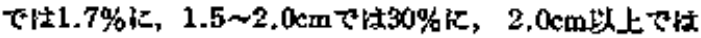

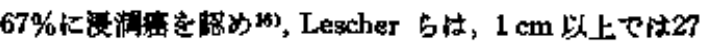

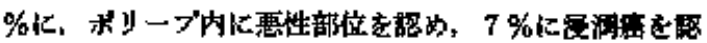

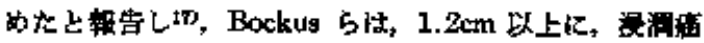

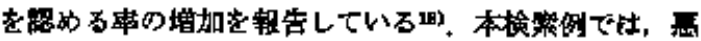

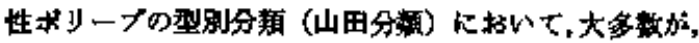

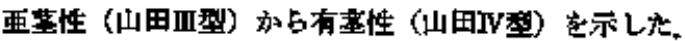

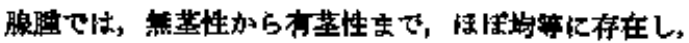
その他のボリーブでは，多くは，篮性であった，この

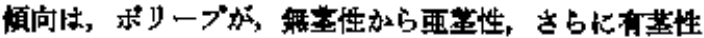

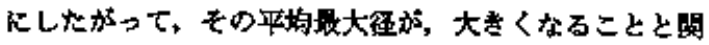
連方あると思かれる。

\section{まとも}

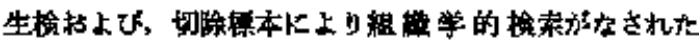
174置３46個の大際ボリープ以ついて梌村した。

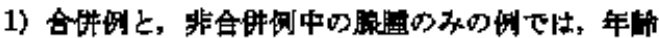
分布のピークの間に10年周の開を斿った。

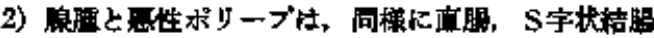

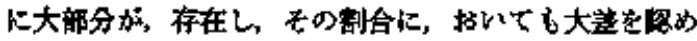

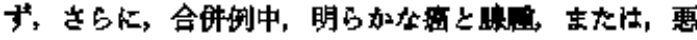

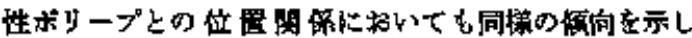
t.

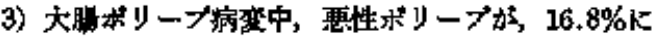

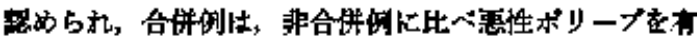

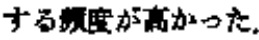

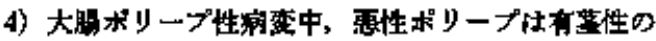

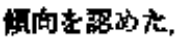

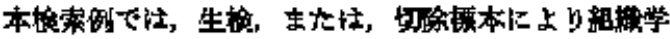

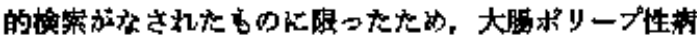

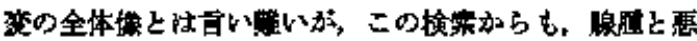
珄ボリープの同には，密接な䖝保があることが推宗され 3.

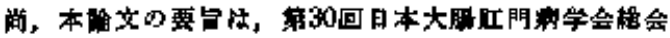
炕て屏表した。

\section{文 音}

1) Morson, B. C. : Precancerous and early malignant lesions of the large intestine. Br. J. Surg. 55 : 725, 1968.

2) Helwig, E. B. : Adenomas and the pathogenesis of cancer of the colon and rectum. $D$ is.Colon \& Rectum. 2 :5, 1959.

3) Spratt, J. S. Jr, Ackerman, L. V., Moyer, C. A. : Relationship of polyps of colon to colonic cancer Ann. Surg. 148 : 682, 1958.

4) Castleman, B., Kriskstein, H. L. : Do adenomatous polype of the colon become malignant? N. Engl. J. Med. 267 : 469, 1962.

5) Miller, C. J., et al. : The Value of procto scopy as a routine examination in preventing death from cancer of large bowel, N. Y. State. J. Med. $50: 2023,1960$.

6) Buie, L. A., et al. : Soltitary adenomata of the rectum and lower signoid. Trans Am. Proct. Soc. $36: 57,1935$.

7) Morson, B. C. : Precancerous condition of the large bowel, Froc. R. Soc. Med. 64 : 959, 1971.

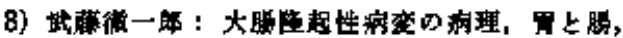
$8: 739,1973$.

9) Buie, L. A., et al. : A re-evaluation of the nalignant potential of colerectal adenomas. 
Surg. Gynecol. Obstet., $119: 867,1964$.

10) Jacktnan, R. J., Mayo, C. W.: The adenoma-carcinoma sequence jn cancer of the colox. S. G. O., $93: 327,1951$.

11) Ekelund,G., et al. : Histopathological analysis of benign polyps in the patient with carcinoma of the colon and rectum. Gut. 15: $654,1974$.

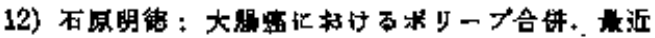
医学, $30: 1639,1975$.

13) Moertel, C. G., et al. : Multiple carcinomas of the large intestine Review of liturature and study of 261 cases. Gastroenterology. 34 : 85, 1958 ,

14) Gilbentsen, V. A.,et al. : Adenacarcinoma of large bowel ; 1340 cases with 100 percent follow up. Surgery, $46: 1027,1969$.

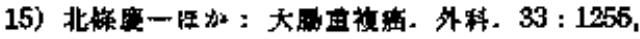
2971.

16) Spratt, J. S. J. : Adenomatous polyps of the rectum and colon. (panel discussion) Dis. Colon \& Rectum. 8: 85, 1965.

17) Lescher, T. C., et al. : Histopathology of the lacger colonic polyps. Dis. Colon \& Rectum. $10: 118,1967$.

18) Bockus, H. L., et al. : Adenomatous polyps of coinn and rectum : Its relation to carcinoma. Gastroenterology. 41 : 225, 1961.

(暗和52年 4 月30日受漹) 
cteristic granuloma, fissuring, giant cells and etc.

Although postoperative course was good after the first operation, rectal lesion was not jmproved and anal fistula still remained.

2nd Operation :

Removal stumpf including anal lesion was perormed on February, 1975 (10 months later). Postoperative course was uneventful showing no evidence of recurrence for more than 3Iyears after 1st operation.

\title{
2. Polyps of the Colon and Rectum: Some Aspects in Patients with Frank Colorectal Carcinoma
}

\author{
S. Iwasa, S. Kobayashi, T. Kasugai and K, Kato \\ Departments of Interngl Medicine and Surgery, Aidui Cancer \\ Center Hoapital, Aichi
}

346 polypoid lesions of the colon and rectum in 174 patients were histologically analysed in either biopsy or surgical specimens at the Aichi Cancer Hospital during the period 1965 to 1973 . Polyosis and pseudopolyps in ulcerative colitis were excluded from this study.

There were 108 men and 66 women. Of these; 73 patients had a frank colorectal carcinoma of which the highest incidence was noted in the seventh decade of life. The age peak in 101 patients without frank colorectal carcinoma was noted in the fifth decade of life and in 44 patients with adenoma alone was noted in the fifth decade of life. The vast majority of edenomas and malignant polyps were found in the sigmoid colon and the rectum. In patients with fratk colorectal carcinoma coexistent adenomas and malignant polyps were similarly distributed in the colon and rectum. The incidence of malignant polyps was 19 percent in 206 polyps of 73 patients with frank colorectal carcinoma while being 13.4 per cent in 141 polyps of 101 patients unessociated with frank colorectal carcinoma. In general, malignant polyps were more to be pedunculated than sessile.

It is conceivable from this study that there is a probable relationship among adenoma, malignant polyp and carcinome of the colon and rectum.

\section{Pathological Study of Rectal Carcinoma (On Retrograde Spread of Rectal Carcinoma in Rectal Wall)}

\author{
N. Kobayashi \\ Department of Surgery, Tokyo Medical College, Tokyo \\ (Director: Prof, Koreyoshi Makino)
}

The present study was made to determine the area to be resected in curative resection 